\section{One-Step Procedure With Isopropanol}

\section{To the Editor:}

In the article by Champagne, Fussell, and Scheifele ${ }^{1}$ the conclusion was made "that two-phase antisepsis using isopropanol followed by $\mathrm{CH}$ is a more effective preparation for blood culture in neonates than is $\mathrm{CH}$ alone." However, they failed to include a control using isopropanol alone although acknowledging that isopropanol is an "excellent antiseptic in its own right." It may be possible that the results ascribed to the two-phase procedure are actually due to the isopropanol alone. Inclusion of this control could have answered this question and possibly indicate that a one-step procedure with the isopropanol would be equivalent to the results presented.

\section{REFERENCE}

1. Champagne S, Fussell S, Scheifele: Evaluation of skin antisepsis prior to blood culture in neonates. Infect Control 1984 5:489-491.

Charles M. Bump, PhD St. Joseph Hospital Flint, Michigan

The authors of the article were invited to respond.

Our principal objective was to compare the skin antisepsis regimen used in our nursery (Hibitane tincture) with recommended regimens (Isopropanol followed by Hibitane tincture or Povidone-iodine solution). ${ }^{1}$ Our observations showed the recommended two-phase regimens to be superior. Because the Hibitane preparation contains $70 \%$ ethanol, we thought it unlikely that Isopropanol used alone would prove satisfactory. Indeed, in eight infants whose forearm skin was colonized with Staphylococcus epidermidis, we found that a 60second application of Isopropanol $70 \%$ de-germed the skin surface of only three. In the remaining five patients, the density of surface bacte- ria was reduced by this treatment but continued to exceed $10^{2}$ organisms per $\mathrm{cm}^{2}$. This result was so like that obtained with Hibitane tincture that we rejected alcohol antisepsis alone as an effective means of preparing infants' skin for blood culture. We are not aware of any authoritative body which has sanctioned alcohol antisepsis for this purpose although we do not deny its usefulness in other circumstances.

\section{REFERENCE}

1. NCCLS Subcommittee for the Standardization of Blood Collection Procedu res in Hematology: Biochemistry and Serolog? 1980 ASH-3 Stamlard Procedures for the Collection of Diagnosic Blood Specimen by Venipuncture. Villona. PA. National Committee for Clinical Laboratory Standards, 1980.

\section{Sylvie Champagne, MD}

David Scheifele, MD

Faculty of Medicine

Department of Paediatrics BCs Children's Hospital Vancouver, BC, Canada

\section{Hepatitis B Vaccine for Health Care Workers}

The advent of a vaccine for protection against Hepatitis $B$ virus infection offers the potential for decreased morbidity and mortality among health care workers. At St. Joseph's Hospital, a 282-bed non-profit community hospital in Elmira, New York, our Infection Control Committee has recommended the use of this vaccine by high-risk employees. ${ }^{1,2} \mathrm{~A}$ variety of approaches to the distribution and financing of hepatitis $B$ vaccine has been offered in the literature. ${ }^{3}$

In September 1983, our hospital administration and Occupational Health Service adopted a compromise plan in which all employees would be offered vaccine, provided one-quarter of the total cost of the three injections $(\$ 103.80)$ was paid by each employee desiring vaccination. An educational series regarding risks and sequelae of hepatitis $\mathbf{B}$ infection vs. vaccinations was already in progress.
Over the first 10 months of the program, only 7 of 1,098 employees (5 of 303 high-risk employees) paid the $\$ 25.95$ and received their vaccine (3 laboratory technicians, 1 pathologist, 1 ER physician, 1 environmental service employee, and 1 laboratory manager). A pre-vaccination screening program for immunity to hepatitis B was not offered, as the cost of screening tests would have been $\$ 15$, and our expected incidence of immunity was less than $20 \%$, even among high-risk employees. ${ }^{4}$

Our Infection Control Committee then designed and mailed a brief questionnaire to the 303 high-risk employees, seeking the reasons for such a poor response to the program. Results among 100 responders were as follows:

Hepatitis B Vaccine Survey As you know, the Hospital's policy is to offer Hepatitis B Vaccine to all employees at a cost of $\$ 25.95$. Employees such as yourself, who are in what is characterized a high-risk group, should seriously consider receiving the vaccine. We ask your assistance by completing this brief survey.

1. Do you desire to obtain the Hepatitis B Vaccine?

Yes 22 No 78

2. If you checked No, please explain:

10 I am afraid of injections.

$\underline{25}$ I do not trust the vaccine.

38 I'm concerned about the after-effects.

68 I can't afford it.

36 I think there should be a screening first.

22 I don't think I need the vaccine.

Our hepatitis B vaccine program has since been revised, and no longer requires a monetary outlay by participants. Sixty-one additional employees (58 in the high-risk category) were vaccinated over the ensuing 3-month period. Our educational efforts, aimed primarily at high-risk personnel, continue. 